\title{
TOWARD CONSISTENT FATIGUE CRACK INITIATION CRITERIA FOR 304L STAINLESS STEEL UNDER MULTI-AXIAL LOADS
}

\author{
Bai-Mao LEI ${ }^{1,2}$, Van-Xuan TRAN ${ }^{3,4, *}$, Saïd TAHERI ${ }^{3,4}$, Jean-Christophe LE ROUX ${ }^{5}$, \\ François CURTIT ${ }^{5}, \mathrm{Mi} \mathrm{HE}^{6}$, Li WAN ${ }^{2}$, and $\mathrm{Yu} \mathrm{ZHOU}^{2}$ \\ ${ }^{1}$ China CEPREI Laboratory, China \\ ${ }^{2}$ Institute of Nuclear and New Energy Technology, Tsinghua University, China \\ ${ }^{3}$ EDF - R\&D, Département Analyses Mécaniques et Acoustique, France \\ ${ }^{4}$ LaMSID, UMR EDF-CNRS-CEA 2832, France \\ ${ }^{5}$ EDF - R\&D, Département Matériaux et Mécanique des Composants, France \\ ${ }^{6}$ EDF - Asia Pacific Branch - China Division R\&D Center, China \\ ${ }^{*}$ Corresponding author. Email address: van-xuan.tran@edfenergy.com
}

\begin{abstract}
In this paper, a new framework to correlate the experimental data of the 304L stainless steel under multiaxial fatigue loadings using a reference curve identified from the experimental data of standard uni-axial fatigue tests is proposed. A total of 15 available fatigue crack initiation criteria are tested on the experimental data of 225 fatigue tests collected from EDF (formerly known as Electricity of France), its partners and the literature. Based on the computational results, it appears that most of the crack initiation parameters of the 15 available criteria can be used to correlate the experimental fatigue data for the 304L stainless steel under uni-axial loadings. Under bi-axial loadings, most of these parameters appear not to be quite consistent. Two criteria are modified in order to better correlate the experimental fatigue data under bi-axial loads. A simple statistical approach to evaluate the consistency of the crack initiation criteria is also proposed. Based on the limited available experimental fagtigue data and within the new framework in this paper, the computational results show that the equivalent crack initiation parameters of the Varvani-Farahani's criterion, the modified Lagoda-Macha-Sakane's criterion and the modified VarvaniFarahani's criterion can be used to correlate the experimental fatigue data under both uni-axial and biaxial loadings for the 304L stainless steel. These criteria may be used to predict the fatigue life of the material under different loading and testing conditions by using the experimental data from the standard uni-axial fatigue tests.
\end{abstract}

\section{INTRODUCTION}

Some 304L stainless steel pipes of cooling systems in nuclear power plants are subjected to cyclic thermo-mechanical loadings during their lifetime. Thermal fatigue occurs and induce in-service damage which has led to shallow cracks in these industrial components [Maillot et al., 2005]. The cyclic thermal stress in the pipe wall can be generally considered as bi-axial tension/compression due to the fact that the radial stress is negligible as compared to the axial and circumferential stresses for a long thin pipe [Kamaya and Taheri, 2008]. It should be noted that most of the available experimental fatigue data for the 304L stainless steel are under uni-axial loading conditions. Only few available experimental fatigue data are under bi-axial tension/compression loading conditions due to the fact that the multi-axial fatigue tests are much more complicated and costly than the standard uni-axial fatigue tests.

Ideally, consistent crack initiation parameters will be identified to be able to predict the fatigue life of the material under different loading and testing conditions by using only the experimental data from the standard uni-axial fatigue tests. In this paper, a new framework is proposed to identify the consistent crack initiation criteria for the 304L stainless steel under bi-axial tension/compression loading conditions. 


\section{METHODOLOGY FOR CORRELATION OF EXPERIMENTAL FATIGUE DATA}

\section{Generalities On Crack Initiation Criteria}

Under cyclic loads, a general crack initiation criterion for fatigue endurance limit can be written as

$$
\Psi(\boldsymbol{\sigma}, \boldsymbol{\varepsilon}, \boldsymbol{n} \ldots)<B
$$

where $\Psi$ represents the equivalent crack initiation parameter and $B$ represents the material property which can be interpreted as the critical value of the equivalent parameter $\Psi$. In Eq. (1), $\sigma$ and $\varepsilon$ represent the applied stress and strain tensors, respectively, and $\boldsymbol{n}$ represents the normal vector of the plane passing through the material point of interest. For the criteria based on the critical plane concept, $\boldsymbol{n}$ represents the critical plane orientation. For this kind of criterion, if Eq. (1) is satisfied, the material point under consideration has no risk of fatigue failure and vice versa.

In this study, the original crack initiation criterion for fatigue endurance limit shown in Eq. (1) is transformed into a damage accumulation criterion as

$$
\Psi(\boldsymbol{\sigma}, \boldsymbol{\varepsilon}, \boldsymbol{n} \ldots)=f\left(N_{f}\right)
$$

where $f\left(N_{f}\right)$ represents a reference "equivalent crack initiation parameter $\Psi$-fatigue life $N_{f}$ " curve. Note that Eq. (2) can be regarded as a general form of the available crack initiation criteria. Particularly, if $\Psi$ is an equivalent stress parameter, $f\left(N_{f}\right)$ represents the Wöhler stress-life curve; If $\Psi$ is an equivalent strain parameter, $f\left(N_{f}\right)$ represents the Manson-Coffin strain-life curve. By using Eq.(2), one can identify the fatigue life $N_{f}$ (number of cycles to failure) at the material point subjected to the loading conditions.

\section{Correlation Of Experimental Fatigue Data Using Equivalent Crack Initiation Parameter}

A correlation of the experimental fatigue data using the equivalent crack initiation parameter is made in two steps as follows:

- Step 1: Identify a reference "equivalent crack initiation parameter $\Psi$-fatigue life $N_{f}$ " curve, by using the experimental fatigue data obtained from standard uni-axial tests for the as-received material at the room temperature under fully reversed loadings(without mean stress or strain). For each reference data point, the value of the equivalent crack initiation parameter $\Psi_{r e f}$ is computed. A fatigue reference curve is fitted by the type of reference curve as

$$
\Psi_{r e f}(\boldsymbol{\sigma}, \boldsymbol{\varepsilon}, \boldsymbol{n}, \ldots)=f\left(N_{f}\right)=a+b N_{f}^{c}
$$

where $a, b$ and $c$ are the fitting coefficients to be determined. In Eq. (3), $a$ can be interpreted as an equivalent endurance limit. 
- Step 2: For the experimental data obtained from each test of which the condition is different from the standard test, the value of the equivalent crack initiation parameter $\Psi$ is evaluated. Then, by using the reference "equivalent crack initiation parameter $\Psi$ - fatigue life $N_{f}$ " curve and the evaluated value of the equivalent crack initiation parameter, the predicted fatigue life $N_{f, p r e d}$ is computed. The value of $N_{f, p r e d}$ is then compared with the experimental fatigue life $N_{f, \exp }$.

For each criterion, the consistency of the crack initiation criterion is then quantified by evaluating the difference between the predicted and experimental fatigue lives. Note that the methodology proposed in this section is a new framework to correlate the experimental fagituge data.

\section{EXPERIMENTAL FATIGUE DATA}

Table 1 lists the description of the experimental fatigue data employed in this study. The data were gathered from EDF, its partners and the literature [Colin et al., 2011; Shamsaei et al., 2011; Lagoda et al., 2003; Itoh et al., 1995], which were obtained from standard specimens made of the 304L stainless steel as discussed in details in the corresponding references. It should be emphasized that all these tests are under constant amplitude fatigue loadings. As listed in the table, a total of 225 data sets are used in this study to evaluate the consistency of the crack initiation criteria. Note that one data set is the experimental data obtained from one fatigue test. As shown in Table 1, there are 139 sets of experimental fatigue data under uni-axial tension/compression loads, 69 sets of experimental fatigue data under bi-axial tension/torsion loads and 20 sets of experimental fatigue data under bi-axial tension/compression loads. As shown in the table, most of data was obtained at the room temperature $\left(20^{\circ} \mathrm{C}\right)$ and some data sets were obtained at the high temperature $\left(300{ }^{\circ} \mathrm{C}\right.$ for uni-axial loads and $650{ }^{\circ} \mathrm{C}$ for bi-axial loads). The conclusions on the consistency of the evaluated crack initiation criteria are therefore based on these limited available data sets.

Table 1: Summary of experimental fatigue data.

\begin{tabular}{ccccccc}
\hline \multirow{2}{*}{ Origin } & \multicolumn{2}{c}{$\begin{array}{c}\text { Uni-axial } \\
\text { tension/compression }\end{array}$} & $\begin{array}{c}\text { Bi-axial } \\
\text { tension/torsion }\end{array}$ & $\begin{array}{c}\text { Bi-axial } \\
\text { tension/compression }\end{array}$ & Total \\
\cline { 2 - 6 } & $20{ }^{\circ} \mathrm{C}$ & $300{ }^{\circ} \mathrm{C}$ & $20{ }^{\circ} \mathrm{C}$ & $650{ }^{\circ} \mathrm{C}$ & $650{ }^{\circ} \mathrm{C}$ & \\
\hline EDF and its partners & 48 & 22 & 3 & 2 & $/$ & 75 \\
Colin et al. & 69 & $/$ & $/$ & $/$ & $/$ & 69 \\
Shamsaei et al. & $/$ & $/$ & 10 & $/$ & $/$ & 10 \\
Lagoda et al. & $/$ & $/$ & $/$ & 21 & 20 & 41 \\
Itoh et al. & $/$ & $/$ & 30 & $/$ & $/$ & 30 \\
Total & 117 & 22 & 43 & 23 & 20 & 225 \\
\hline
\end{tabular}

\section{CORRELATION OF EXPERIMENTAL FATIGUE DATA USING AVAILABLE CRITERIA}

In this paper, a total of 15 available crack initiation criteria are evaluated by using the correlation methodology as previously presented. Due to length constraint of the paper, only the results for 2 criteria are presented. 


\section{For Lagoda-Macha-Sakane's Criterion}

Lagoda, Macha and Sakane[Lagoda et al., 2003] considered the maximum normal elastic and plastic strain energy densities on the critical plane and proposed an equivalent crack initiation parameter as follows

$$
\psi=\left(\frac{\Delta \sigma_{\mathrm{n}} \Delta \varepsilon_{\mathrm{n}}}{8}\right)_{\max }
$$

Note that the critical plane is defined as the plane where the crack initiation parameter $\psi$ reaches its maximum value. In Eq. (4), $\Delta \sigma_{\mathrm{n}}$ and $\Delta \varepsilon_{\mathrm{n}}$ represent the amplitude of the normal stress and normal strain on the critical plane, respectively.

Figure 1 shows the correlation of uni-axial and bi-axial experimental data using Lagoda-Macha-Sakane's criterion. As shown in Figure 1(a), most of the experimental fatigue data under uni-axial loading conditions by using the Lagoda-Macha-Sakane's equivalent crack initiation parameter appear to be within the band 2 curves for the data with the fatigue life smaller than $2 \times 10^{4}$ cycles. For the fatigue life higher than $2 \times 10^{4}$ cycles, some important scatter can be noticed. As shown in Figure 1(b), most of the experimental fatigue data under bi-axial loading conditions by using the Lagoda-Macha-Sakane's equivalent crack initiation parameter appear to be quite scattered. Most of the experimental fatigue data obtained from the tests at $650{ }^{\circ} \mathrm{C}$ appear to be above the upper band 4 curve. The use of the LagodaMacha-Sakane's equivalent crack initiation parameter may then lead to over-estimate the fatigue life for bi-axial loads at high temperature.

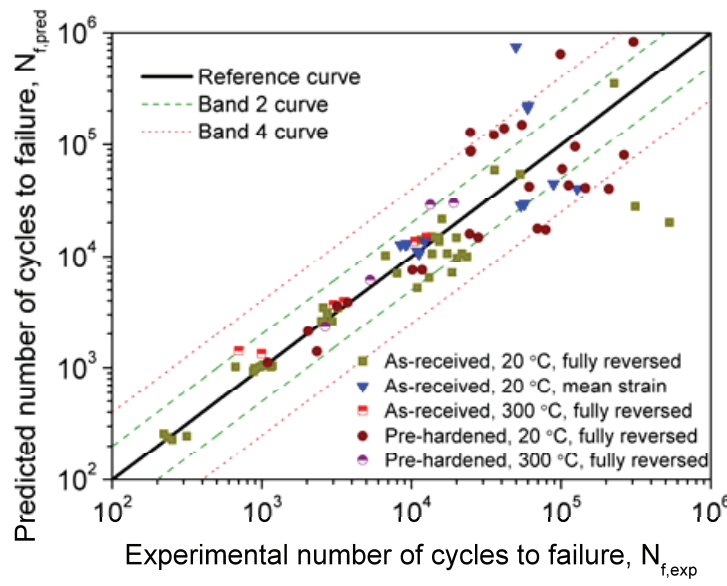

(a)

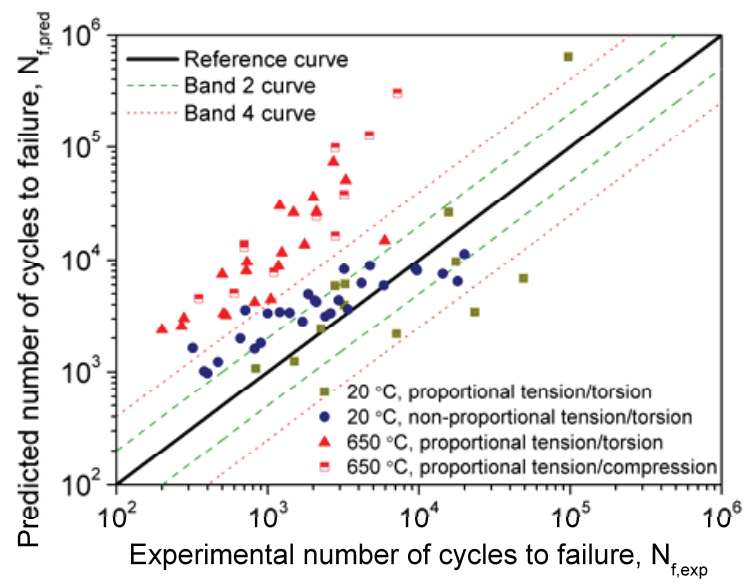

(b)

Figure 1. Correlation of (a) uni-axial, (b) bi-axial experimental data using Lagoda-Macha-Sakane's criterion.

In conclusion, the Lagoda-Macha-Sakane's equivalent crack initiation parameter can be used to correlate the experimental fatigue data under uni-axial low-cycle loading conditions. This parameter seems not to be quite consistent to correlate the experimental fatigue data for the 304L stainless steel under high-cycle uni-axial and bi-axial loading conditions, especially at high temperature. 


\section{For Varvani-Farahani's criterion}

Varvani-Farahani[Varvani-Farahani, 2000] proposed the summation of the normal energy range and the shear energy range computed on the critical plane, which were both divided by the tensile and shear fatigue properties, to be a crack initiation parameter. The Varvani-Farahani's equivalent crack initiation parameter can be expressed as

$$
\psi=\frac{\Delta \sigma_{\mathrm{n}} \Delta \varepsilon_{\mathrm{n}}}{\sigma_{\mathrm{f}}^{\prime} \varepsilon_{\mathrm{f}}^{\prime}}+\left(1+\frac{\sigma_{\mathrm{n}, \text { mean }}}{\sigma_{\mathrm{f}}^{\prime}}\right) \frac{\Delta \tau_{\max } \Delta\left(\frac{\gamma_{\text {max }}}{2}\right)}{\tau_{\mathrm{f}}^{\prime} \gamma_{\mathrm{f}}^{\prime}}
$$

Note that the critical plane is defined as the plane on which the stress and strain Mohr's circles are the largest during the loading and unloading parts of a cycle. In Eq. (5), $\sigma_{\mathrm{n} \text {,mean }}$ represents the mean value of normal stress; $\Delta \tau_{\max }$ and $\Delta \gamma_{\max }$ represent the maximum amplitude of normal stress and shear stress, respectively. Also, $\sigma_{\mathrm{f}}^{\prime}$ and $\tau_{\mathrm{f}}^{\prime}$ represent the fatigue strength coefficients in tension and shear, respectively; $\varepsilon_{\mathrm{f}}^{\prime}$ and $\gamma_{\mathrm{f}}^{\prime}$ represent the fatigue ductility coefficients in tension and shear, respectively.

Figure 2 shows the correlation of uni-axial and bi-axial experimental data using Varvani-Farahani's criterion. As shown in Figure 2(a), most of the experimental fatigue data under uni-axial loading conditions by using the Varvani-Farahani's equivalent crack initiation parameter appear to be within the band 2 curves for the data with the fatigue life smaller than $10^{4}$ cycles. For the fatigue life higher than $10^{4}$ cycles, some important scatter can be noticed. As shown in Figure 2(b), the experimental fatigue data under bi-axial loading conditions by using the Varvani-Farahani's equivalent crack initiation parameter appear to be quite scattered. As shown in the figure, most of the experimental fatigue data for nonproportional tension/torsion loads appear to be outside of the band 4 curves.

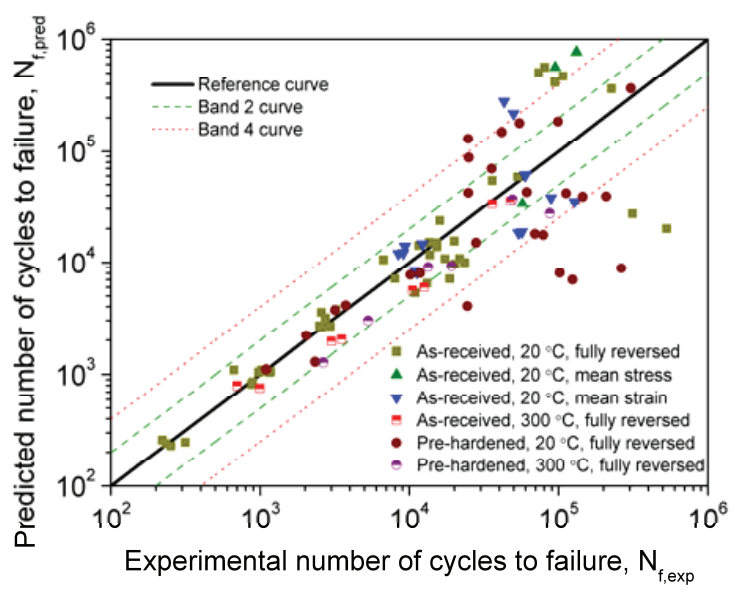

(a)

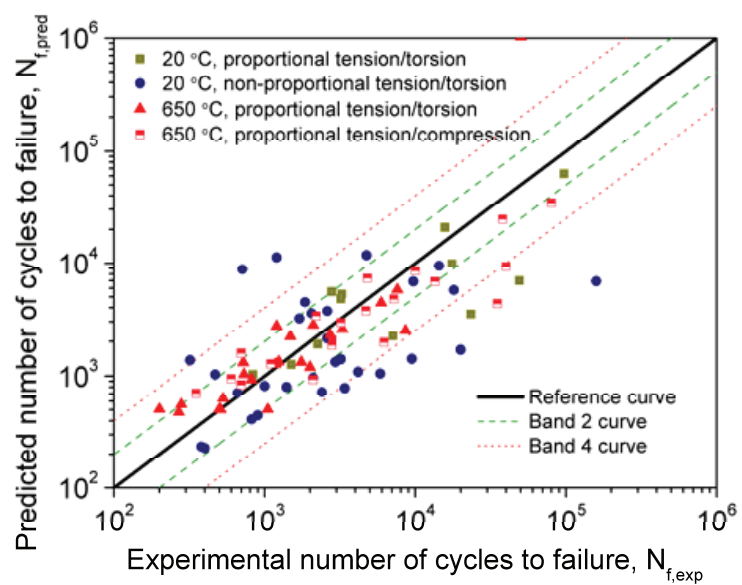

(b)

Figure 2. Correlation of (a) uni-axial, (b) bi-axial experimental data using Varvani-Farahani's criterion.

In conclusion, the Varvani-Farahani's equivalent parameter can be used to correlate the experimental fatigue data under uni-axial low-cycle loading conditions. This parameter seems not to be quite consistent to correlate the experimental fatigue data for the 304L stainless steel under high-cycle uni-axial and biaxial loading conditions, especially for non-proportional tension/torsion loads. 


\section{NEWLY MODIFIED CRACK INITIATION CRITEIRA}

\section{Modified Lagoda-Macha-Sakane's criterion}

As shown in Figure 1(b), the Lagoda-Macha-Sakane's criterion seems to be consistent with the experimental fatigue data at $20^{\circ} \mathrm{C}$. However, it is not consistent with the experimental fatigue data at 650 ${ }^{\circ} \mathrm{C}$. As shown in Figure 2(b), the Varvani-Farahani's criterion seems to be consistent with the experimental fatigue data at $650{ }^{\circ} \mathrm{C}$. Note that in Varvani-Farahani's criterion as in Eq. (5), the normal work term and shear work term are modified by the fatigue strength coefficient $\sigma_{\mathrm{f}}^{\prime}, \tau_{\mathrm{f}}^{\prime}$, and the fatigue ductility coefficient $\varepsilon_{\mathrm{f}}^{\prime}, \gamma_{\mathrm{f}}^{\prime}$. These material coefficients are all dependent on the temperature. Therefore, they may be used to take into account the temperature effects. Based on these remarks, the original Lagoda-Macha-Sakane's crack initiation parameter is modified as

$$
\psi=\left(\frac{\Delta \sigma_{\mathrm{n}} \Delta \varepsilon_{\mathrm{n}}}{8 \sigma_{\mathrm{f}}^{\prime} \varepsilon_{\mathrm{f}}^{\prime}}\right)_{\text {max }}
$$

Figure 3 shows the correlation of uni-axial and bi-axial experimental data using the modified LagodaMacha-Sakane's criterion. As shown in Figure 3, most of the experimental fatigue data under uni-axial and bi-axial loading conditions by using the modified Lagoda-Macha-Sakane's equivalent crack initiation parameter appear to be within the band 2 curves. As compared to Figure 1, the modified Lagoda-MachaSakane's crack initiation parameter appears to correlate the experimental fatigue data better than the original Lagoda-Macha-Sakane's crack initiation parameter. This modified criterion may be a good criterion to describe the fatigue behavior of the 304L steel under bi-axial loading conditions.

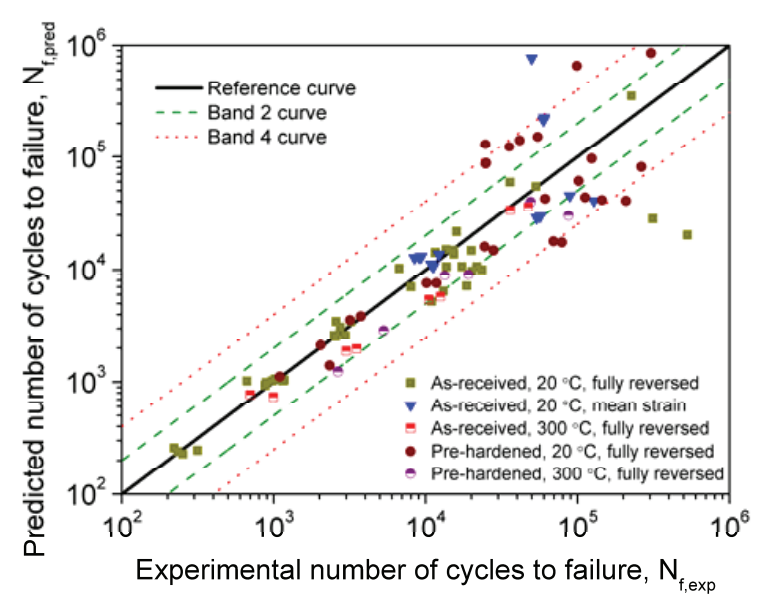

(a)

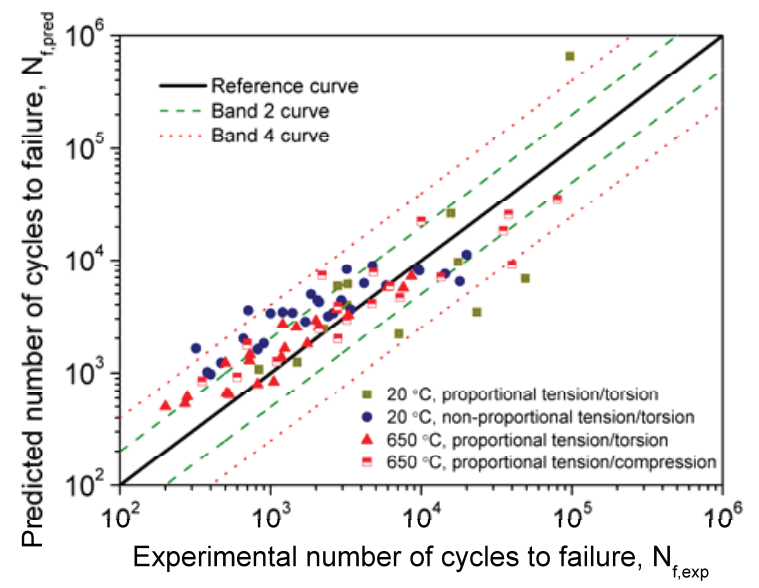

(b)

Figure 3. Correlation of (a) uni-axial, (b) bi-axial experimental data using the modified Lagoda-MachaSakane's criterion.

\section{Modified Varvani-Farahani's criterion}

As shown in Figure 2(b), the Varvani-Farahani's criterion parameter seems to be consistent with the experimental fatigue data except that under non-proportional loadings. Note that the non-proportionality 
induces additional cyclic hardening and may be considered in the term of mean stress modification in the crack initiation criterion. The original Varvani-Farahani's criterion is modified as

$$
\psi=\left[\left(1+\frac{\sigma_{\mathrm{n}, \max }}{\sigma_{\mathrm{f}}^{\prime}}\right) \frac{\Delta \sigma_{\mathrm{n}} \Delta \varepsilon_{\mathrm{n}}}{4 \sigma_{\mathrm{f}}^{\prime} \varepsilon_{\mathrm{f}}^{\prime}}+\frac{\left(\frac{\Delta \tau}{2}+a_{\mathrm{m}} \sigma_{\mathrm{n}, \max }\right) \frac{\Delta \gamma}{2}}{\tau_{\mathrm{f}}^{\prime} \gamma_{\mathrm{f}}^{\prime}}\right]_{\max }
$$

where $a_{\mathrm{m}}$ is the material constant as in the Matake's criterion. Note that the mean stress modification of the shear stress term is actually the same as that in the Matake's criterion. The critical plane is defined as the plane where the equivalent crack initiation parameter $\psi$ reaches its maximum value.

Figure 4 shows the correlation of uni-axial and bi-axial experimental data using the modified VarvaniFarahani's criterion. As shown in Figure 4, most of the experimental fatigue data under uni-axial and biaxial loading conditions by using the modified Varvani-Farahani's equivalent crack initiation parameter appear to be within the band 2 curves. As compared to Figure 2, the modified Varvani-Farahani's crack initiation parameter appears to correlate the experimental fatigue data for the $304 \mathrm{~L}$ stainless steel better than the original Varvani-Farahani's crack initiation parameter. This modified criterion may be a good criterion to describe the fatigue behavior of the $304 \mathrm{~L}$ stainless steel under bi-axial loading conditions.

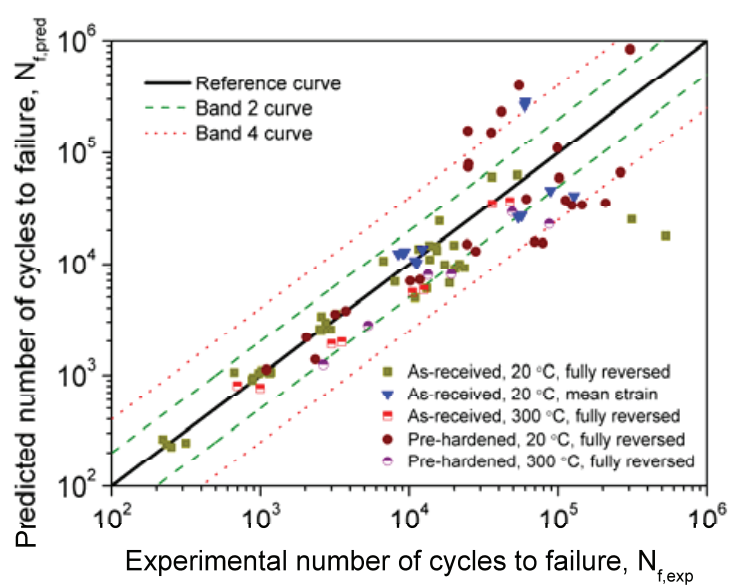

(a)

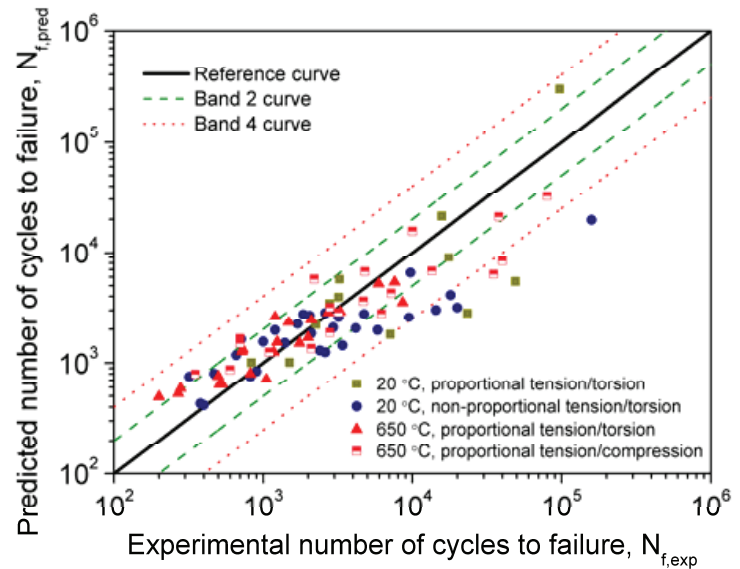

(b)

Figure 4. Correlation of (a) uni-axial, (b) bi-axial experimental data using the modified VarvaniFarahani's criterion.

\section{CONSISTENCY EVALUATION OF CRACK INITIATION CRITERIA}

As discussed in the previous sections, 17 crack initiation criteria in total have been evaluated using the 225 available experimental data sets. The computational results obtained from different criteria are quite different. It is therefore important to characterize the consistency of each criterion in order to examine its applicability to correlate the experimental fatigue data. In this section, a simple statistical approach is proposed.

In reference to a given crack initiation criterion, for each data set, an error index $E I$ of the fatigue life prediction in the logarithmic scale is defined as 


$$
E I=\log \frac{N_{f, \text { pred }}}{N_{f, \exp }}
$$

where $N_{f, \text { pred }}$ and $N_{f, \text { exp }}$ represent the predicted (computational) and experimental number of cycles to failure, respectively. On the reference curve, $N_{f, p r e d}$ equals to $N_{f, \text { exp }}$. This reference curve represents a line with $E I=0$ in the logarithmic scale. The upper and lower band 2 curves correspond to the lines $E I=$ $\log 2$ and $E I=-\log 2$, respectively. Two statistical parameters are then defined, namely, mean value of the logarithmic error index $M V E$ as

$$
M V E=\frac{\sum_{i=1}^{n_{\text {total }}} E I_{i}}{n_{\text {total }}}
$$

and standard deviation of the logarithmic error index $S D E$ as

$$
S D E=\sqrt{\frac{\sum_{i=1}^{n_{\text {total }}}\left(E I_{i}-M V E\right)^{2}}{n_{\text {total }}}}
$$

where $n_{\text {total }}$ represents the number of data points and $E I_{i}$ (with $i=1,2, \ldots n_{\text {total }}$ ) represent the error index of each data set.

In general, a criterion can be considered to be consistent if the absolute value of $M V E$ is small and the data is not very scattered (the absolute value of $S D E$ should be small). For this reason, another simple statistical parameter is used in this paper as $|M V E|+|S D E|$, denoted as the consistency parameter. A criterion providing the smaller values of $|M V E|+|S D E|$ will then be considered to be more consistent to correlate the experimental fatigue data.

Figure 5 shows the statistical description of the results of fatigue life prediction using various crack initiation criteria under uni-axial, bi-axial tension/torsion and bi-axial tension/compression fatigue loading conditions. As shown in Figure 5(a), under uni-axial loading conditions, most of the 17 crack initiation criteria appear to be consistent. It is noted that the values of $M V E$ for all evaluated criteria are smaller than the distance represented by the band 2 curve (of which the values of $M V E$ are $\pm \log 2= \pm 0.3$ ). As shown in Figure 5(b), under bi-axial tension/torsion loading conditions, some of the 17 crack initiation criteria appear to be consistent such as the Glinka Wang Plumtree's, the Varvani-Farahani's, the modified Lagoda-Macha-Sakane's and the modified Varvani-Farahani's criteria. As shown in Figure 5(c), under biaxial tension/tension loading conditions, only the Varvani-Farahani's, the modified Lagoda-MachaSakane's and the modified Varvani-Farahani's criteria appear to be consistent.

In conclusion, while most of the tested criteria appear to be consistent under un-axial loading conditions, only few of them appear to be more applicable for the bi-axial loading conditions. Based on the limited available experimental fatigue data and by using the consistency parameter $|M V E|+|S D E|$, the equivalent crack initiation parameters of the Varvani-Farahani's, the modified Lagoda-Macha-Sakane's and the modified Varvani-Farahani's criteria appear to be consistent to correlate the experimental fatigue data for the 304L stainless steel. By using these parameters, one can predict the fatigue life of the material 
under different loading and testing conditions by using the experimental data from the standard uni-axial fatigue tests.

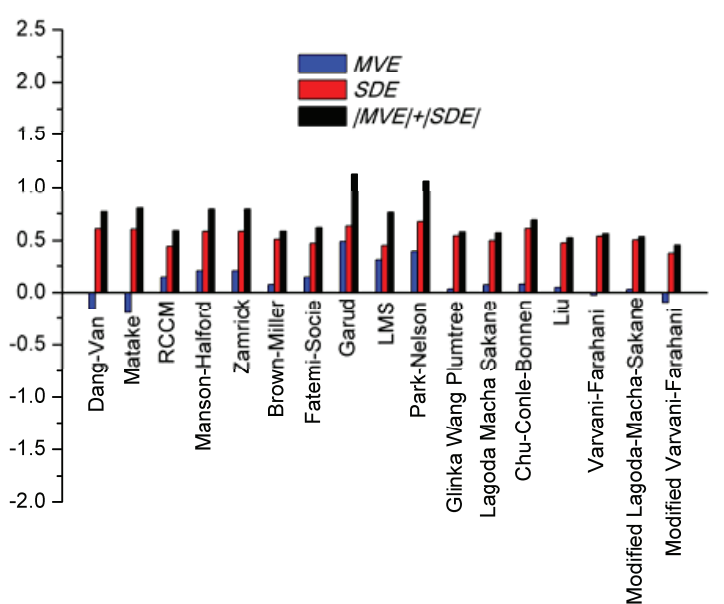

(a)

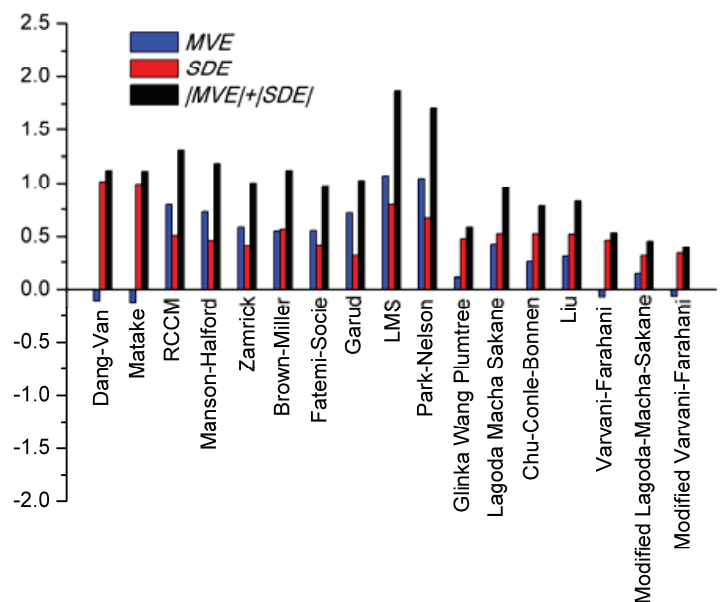

(b)

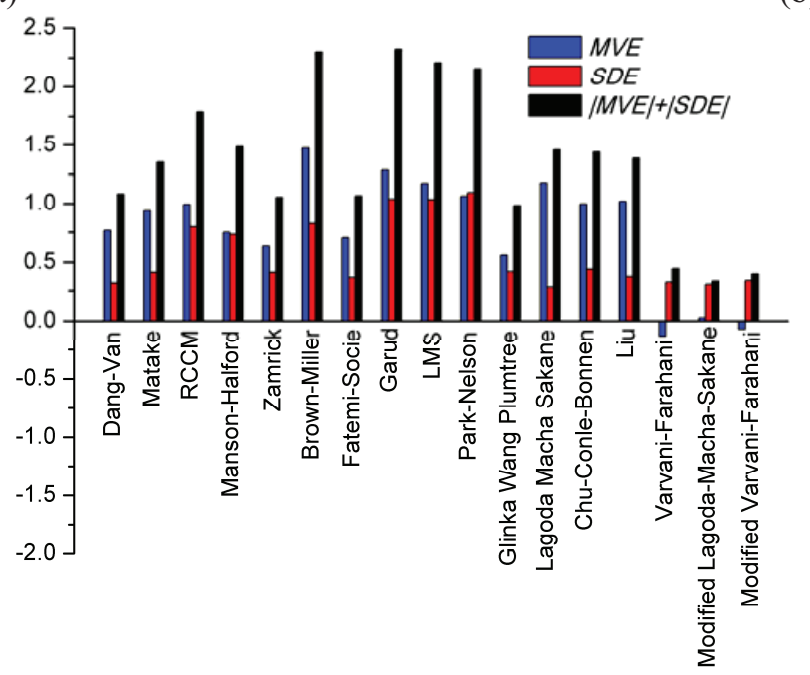

(c)

Figure 5. Statistical description of the results of fatigue life prediction using various crack initiation criteria under (a) uni-axial, (b) bi-axial tension/torsion, (c) bi-axial tension/compression fatigue loads.

\section{DISCUSSIONS}

It should be noted that the experimental fatigue data presented in this study are collected from EDF, its partners and the literature. The testing conditions and specimen geometries from different laboratories may not be the same. Also, the chemical compositions of different 304L stainless steel alloys may be different. The evaluation of the experimental fatigue data which were not obtained under the same loading conditions would include some sources of uncertainty. Also, each criterion tested in this study was originally developed to investigate the fatigue behaviours at different regimes (low cycle fatigue, high cycle fatigue, endurance limit). The use of these criteria without considering their applicability may also lead to the non-consistency of the equivalent crack initiation parameters. Also, the use of the material properties from different references is approximate. The main purpose of this paper is to propose a simple 
$23^{\text {rd }}$ Conference on Structural Mechanics in Reactor Technology Manchester, United Kingdom - August 10-14, 2015

Division III

engineering methodology to identify a consistent crack initiation criterion for a specific material. For this reason, the conclusion on the consistency of the equivalent crack initiation parameters of the VarvaniFarahani's, the modified Lagoda-Macha-Sakane's and the modified Varvani-Farahani's criteria should be considered only within the new framework proposed in this particular study.

Regarding the statistical analysis, only limited numbers of experimental fatigue data sets are available in this study, especially for the bi-axial tension/tension loading conditions. From the statistical point of view, the relatively small number of the experimental fatigue data sets can affect the conclusion on the criterion consistency. This kind of study should be therefore regularly updated if more experimental fatigue data sets are available. Also, the choice of a quantitative parameter $|M V E|+|S D E|$ for the consistency evaluation in this study is simply for convenient discussion. Different choices of the consistency parameter will depend on the application of the study under investigation and may lead to different conclusions on the criterion consistency.

\section{CONCLUSION}

In this paper, a new framework to correlate the experimental data of the 304L stainless steel under multiaxial fatigue loadings using a reference curve identified from the experimental data of standard uni-axial fatigue tests is proposed. Based on the computational results, it appears that most of the crack initiation parameters of the 15 available criteria can be used to correlate the experimental fatigue data for the 304L stainless steel under uni-axial loadings. Under bi-axial loadings, most of these parameters appear not to be quite consistent. Two criteria are modified in order to better correlate the experimental fatigue data under bi-axial loads. A simple statistical approach to evaluate the consistency of the crack initiation criteria is also proposed. Based on the limited available experimental fatigue data, the computational results show that the equivalent crack initiation parameters from the Varvani-Farahani's criterion, the modified Lagoda-Macha-Sakane's criterion and the modified Varvani-Farahani's criterion can be used to correlate the experimental fatigue data under both uni-axial and bi-axial loadings for the $304 \mathrm{~L}$ stainless steel. These criteria may be used to predict the fatigue life of the 304L stainless steel under different loading and testing conditions by using the experimental data from the standard uni-axial fatigue tests.

\section{REFERENCES}

Maillot, V., Fissolo, A., Degallaix, G. and Degallaix, S. (2005). "Thermal fatigue crack networks parameters and stability: an experimentalstudy," International Journal of Solids and Structures, 42: 759-769

Kamaya, M., and Taheri, S. (2008). "A study on the evolution of crack networks under thermal fatigue loading," Nuclear Engineering and Design, 238: 2147-2154

Colin, J., Fatemi, A., and Taheri, S. (2011). "Cyclic hardening and fatigue behavior of stainless steel 304L," Journal of Materials Science, 46(1): 145-154.

Shamsaei, N., Fatemi, A., and Socie, D. F. (2011). "Multiaxial fatigue evaluation using discriminating strain paths," International Journal of Fatigue, 33(4): 597-609.

Lagoda, T., Macha, E., and Sakane, M. (2003). "Estimation of high temperature fatigue lifetime of SUS304 steel with an energy parameter in the critical plane," Journal of Theoretical and Applied Mechanics, 41(1): 55-73.

Itoh, T., Sakane, M., and Ohnami, M. (1995). "Nonproportional low cycle fatigue criterion for type 304 stainless steel," Journal of Engineering Materials and Technology, Transactions of the ASME, 117(3):285-292.

Varvani-Farahani, A. (2000). "New energy-critical plane parameter for fatigue life assessment of various metallic materials subjected to in-phase and out-of-phase multiaxial fatigue loading conditions," International Journal of Fatigue, 22(4):295-305. 